\title{
EVIDENCE-BASED PRACTICE: CHALLEGES TO MEDICAL REHABILITATION PRACTITIONERS
}

In recent times, the medical rehabilitation practitioners especially physiotherapy profession has undergone a period of change as a result of pressure from different health professional groups, and in order to meet this threat, physiotherapists have been encouraged to prove the effectiveness of their clinical interventions by gaining scientific evidence through a process known as evidencebased practice (Sackett et al, 2000).

Evidence-based practice (EBP) is an outgrowth of evidence-based medicine. Evidence-based medicine was initially defined as the conscientious, explicit and judious use of current and objective best evidence in making sound and effective clinical decision about the diagnosis, treatment and prognosis of individual patients (Sackett et al, 2000). It represents a radical shift from a paradigm of knowledge that was based on autonomy and clinical experience. Evidence-based medicine initially emphasized the importance of evaluating a large body of medical literature and disseminating the most valid and important findings to the medical community. Evidence-based practice broadened this conceptuation and is now defined to also include the integration of individual clinical expertise, individual patient preferences, and the best available external evidence from systematic research in order to best guide clinical decision making.

Evidence-based practice utilizes a structured approach involving 5 key steps which includes;

- Convert the need for information into a clinically relevant answerable question

- Efficiently find the best evidence

- Critically appraise the evidence

- Applying critically appraised evidence to EBP

- Evaluating and documenting the effect of EBP on clinical outcomes using objective outcome measures

Within the international physiotherapy community, there is a growing acceptance of this approach, which is referred to as Evidence-based physiotherapy (Sackett et al, 2000).

Evidence-based physiotherapy has become feasible with the enormous increase in recent years, in the volume and accessibility of high quality clinical research. The demand for and interest in applying evidence to physiotherapy practice has grown in the past decade as demonstrated by the publication of a series of systematic reviews in the October 2001 special issue of Physiotherapy and other articles related to evidence in practice (Ciccone, 2003). Studies have shown that clinically relevant researches as well as clinical expertise are important components of EBP, and that identification and application of patient's preferences should be part of clinical decision-making (Sackett et al, 2000). The concept of EBP marks a shift among health care professionals from a traditional emphasis on action based on the "opinions of authorities to guide clinical practice" to an 
emphasis on "data based clinically relevant studies and researches" (Guyalt et al, 2000). Peckham (1991) reported that strongly held views based on belief rather than sound information still exerts much influence on health care.

To effectively apply evidence in practice, in addition to skill in taking a history, conduction of clinical examination and screening, determining appropriate options for intervention, Guyalt et al (2000), maintained that a clinician must have the ability to identify grasps in knowledge, formulate clinically relevant questions, conduct an effective literature search, apply rules of evidence including a hierarchy of evidence to determine the validity of studies, apply the literature findings appropriately to the patient's problem and understand how the patient's value affects the balance between the potential advantage and disadvantage of the available management options and appropriately involve the patient in the clinical decision making. Although the importance of evidencebased practice was identified decades ago, it has gradually been adopted by the physiotherapy profession. There are a number of challenges for physiotherapists who are attempting to use research to aid clinical decision-making (Harrison, 2002). Most challenges can be grouped under one of three areas, research methods, clinician's skill and administrative factors (Harrison, 2002).

The goal of EBP is to use the knowledge created by scientific research in practice (Harrison, 1996) and this cannot happen without the clinicians, who are the principal stake-holder. The promotion and development of a clinical culture that understands research, values the evidence generated by that research and demands to be informed is essential. However, clinicians are often unclear as to the meaning of EBP and they may not understand the types of research that constitute high quality evidence (Morris, 2003). Physiotherapists often have difficulty applying research findings to individual patients and are unclear as to whether high quality evidence exists to support or refute therapeutic intervention (Jette et al, 2000).

Much of what physiotherapists do awaits definitive research to establish its efficacy (Morris, 2003). In many instances, there is little evidence to support or refute current practices. Clinicians' negative attitudes about research further compound the difficulties. The transition to EBP may not readily occur if clinicians do not know about the evidence, do not understand it, believe it, or know how to apply the findings to clinical practice.

The importance of EBP to physiotherapy cannot be over emphasize, these includes;

- Improves quality of patients care

- Reduces cost

- Guide resources allocation

- Educates patients

- Assist clinical decision making

Some of the major barriers to EBP are;

- Insufficient time due to large patients load on daily basis 
- Lack of information resources, e.g. unavailability of Internet facility

- Inability to apply research findings to individual patients

- Lack of research skills

- Lack of interest

- Poor ability to critically appraise the literature.

In conclusion, the challenges and barriers to EBP are enormous but we must be ready to face them taking into consideration our professional peculiarity and the environment in which we practice. With determination we shall overcome the barriers because incorporating EBP to our practice is not only a plus for us or our profession but to entire human race. Therefore Physiotherapy training institutions, hospitals and clinics, Medical Rehabilitation Therapy Board, Nigerian Society of Physiotherapy and other professional associations in the medical sector must encourage and support this phenomenon as it is been done in the developed countries.

\section{References}

- Ciccone CD (2003). Clinical questions: Does ambulation immediately following an episode of deep vein thrombosis increases the risk of of pulmonary embolism?. Physical Therapy 82: 84-88

- Guyalt GH, Haynes RB, Jaeschke RZ (2000). Evidence based medicine principles for applying the users to patient care. JAMA 308: 1290-1296.

- Harrison M (2002). Evidence-based practice: Challeges in practice based on evidence. Physiotherapy Theory \& Practice 25: 80-1 12

- Jette DU, Bacon K, Batty C (2003). Evidence-based practice: Beliefs, attitudes, knowledge and behaviors of Physical Therapists. Physical Therapy 83: 786-805

- Morris LR (2003). Facilitating a culture of evidence in Physiotherapy practice. Health policy.

- Peckham MC (1991). Research for health. Journal of the National Scheme 22: 102-107.

- Sackett DL, Strauss SE, Richardson WS (2000). Evidence-based medicine: How to practice and teach evidence-based medicine. Churchill Livingstone Edinburgh, Scotland: 1-120

SRA Akinbo $P h D$

Editor-in-Chief \& Chairman Editorial Board, NJMR

http://mrtbjournal.org/index.php/njmr/issue/current/showToc 